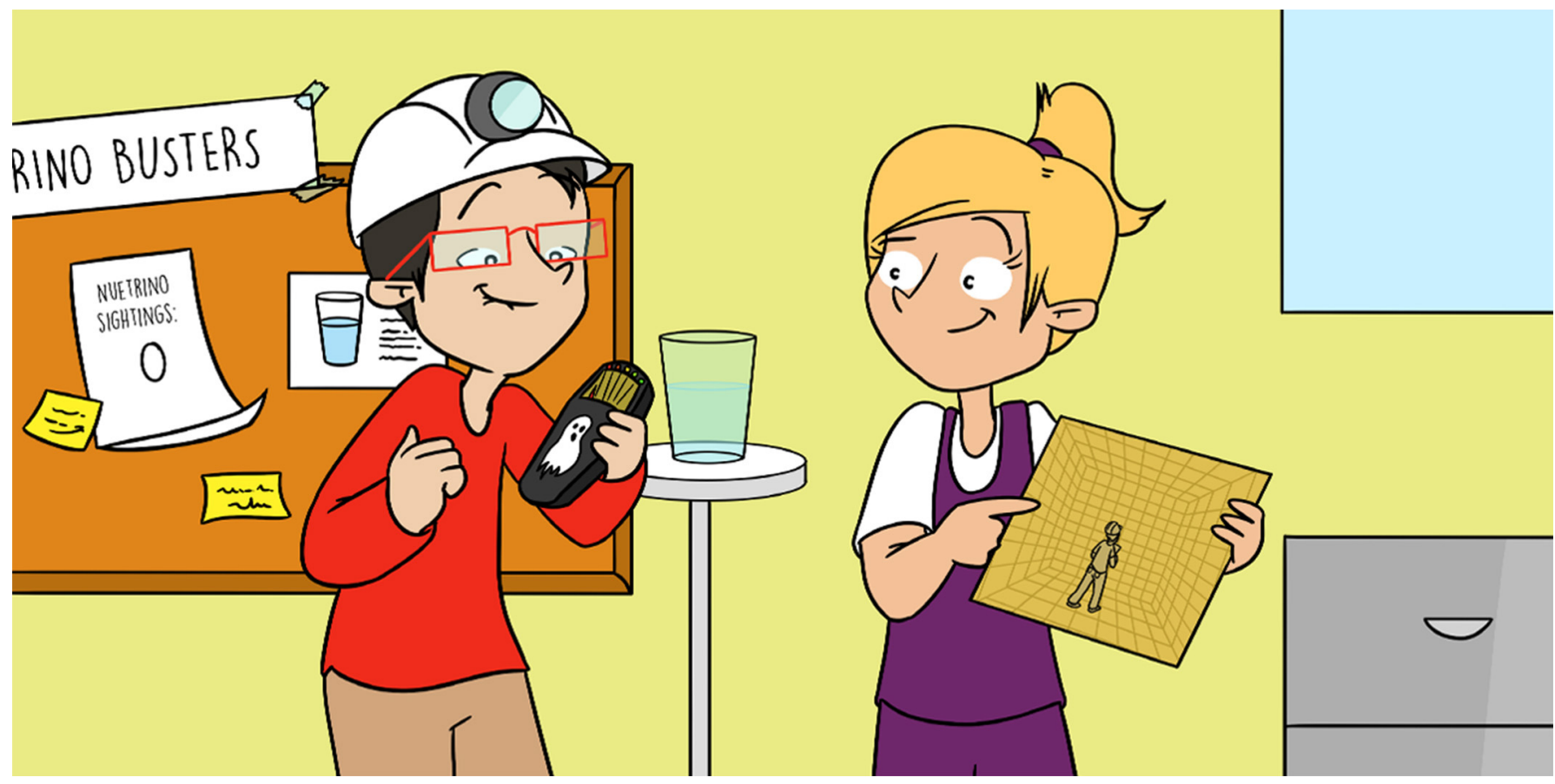

\title{
DETECTING GHOSTLIKE NEUTRINOS: TINY MESSENGERS FROM THE UNIVERSE
}

\section{Milind Vaman Diwan ${ }^{1 *}$ and Karen McNulty Walsh ${ }^{2}$}

${ }^{1}$ Physics Department, Brookhaven National Laboratory, Upton, NY, United States

${ }^{2}$ Media \& Communications Office, Brookhaven National Laboratory, Upton, NY, United States

YOUNG REVIEWERS:

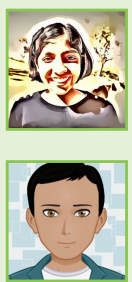

ANUSHKA

AGE: 12

RIK

AGE: 13
Neutrinos are tiny, subatomic particles. Billions of them pass through us every second-mostly coming from our sun. But unlike the sunlight we can easily see, neutrinos are very hard to detect. To "see" them, we have to build really big detectors and block out signals from any other particles. Scientists do that by building neutrino detectors deep underground. In this article you will learn how these giant detectors can help decode the "messages" these ghostlike particles deliver about the makeup and history of the stars, galaxies, and the universe.

\section{INTRODUCTION}

Neutrinos are tiny, subatomic particles that seem almost like ghosts. Trillions of them pass through us every second without leaving a trace! They mostly come from the nuclear reactions that power our sun. But unlike the sunlight we see and feel on our faces, these ghostlike particles do not often interact with ordinary materials. To 
Figure 1

Decay of a radioactive atom, potassium-40which is found in bananas and has 40 protons ( $p$ ) and neutrons ( $n$ ) in its nucleus -into calcium-40, plus an electron $\left(\mathrm{e}^{-}\right)$and $a$ neutral "ghostlike" messenger particle called a neutrino (Greek letter: v, pronounced "nu"). Understanding the behavior of neutrinos may help reveal important secrets of the universe. Credit: Tiffany Bowman, Brookhaven National Laboratory.

\section{RADIOACTIVE}

\section{DECAY}

The nuclei of many atoms are unstable, and over time they break apart releasing energetic particles, such as alpha, beta, and gamma rays. This process of breaking apart is called radioactive decay.

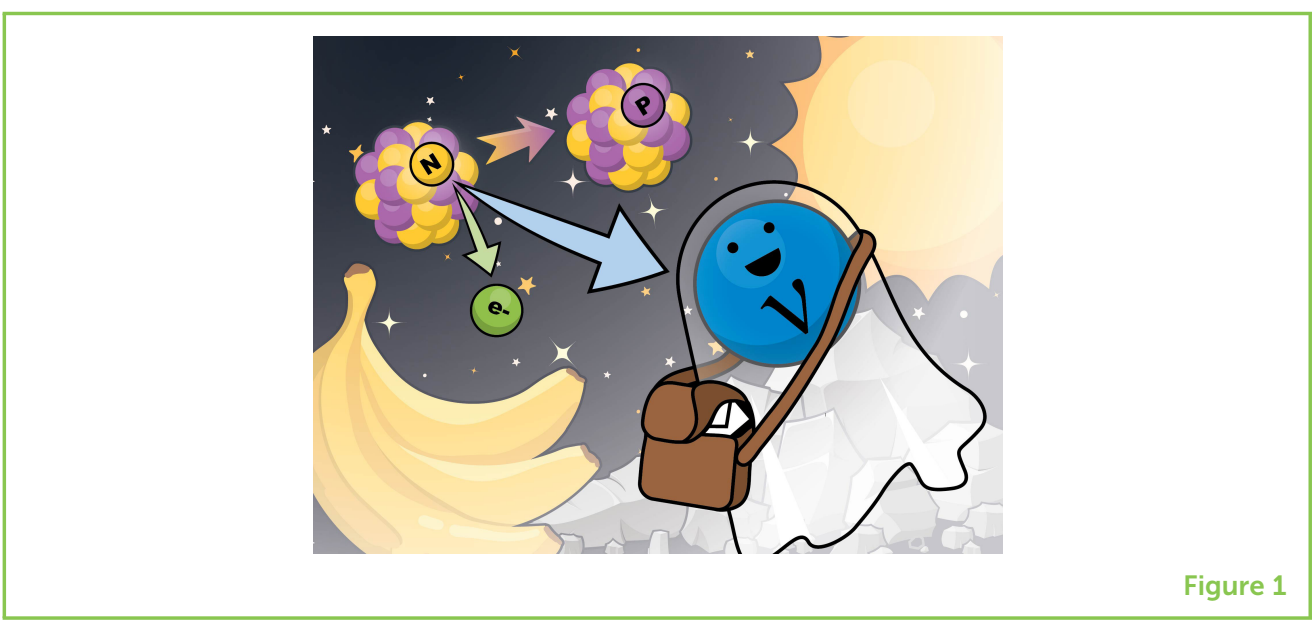

"see" neutrinos, scientists build really big, sensitive detectors, often deep underground. The matter that makes up Earth helps shield out signals from other particles, so that the signals of neutrinos' rare interactions stand a chance of being detected. These giant detectors are helping scientists to pick up the "messages" that these ghostlike particles carry. When scientists decode the messages, they learn about the connection between neutrinos and the makeup and history of the stars, galaxies, and the universe.

\section{WHAT IS A NEUTRINO?}

Neutrinos are very light particles with no electric charge. They are emitted when unstable atomic nuclei decay. Such radioactive decay happens all around us. Minerals in rocks and even potassium in bananas decay to produce neutrinos (see Figure 1). But far more neutrinos come from the nuclear reactions in the sun than from any other source.

No matter what their source, neutrinos are completely harmless because they do not stick together or interact with much of anything. They stream from the sun and outer space and pass right through ordinary matter-even our own bodies and Earth itself-without us ever knowing. In that way, they seem a bit like ghosts-there, and yet invisible. But because neutrinos carry information about what is happening inside the hearts of stars and galaxies, scientists want to know a lot more about them. Neutrinos may even help us understand what was going on when our universe first formed nearly 14 billion years ago!

\section{HOW TO CATCH A GHOSTLIKE PARTICLE}

Neutrinos rarely interact with ordinary matter, so they are very hard to detect. If you place a glass of water on a table, a trillion neutrinos 


\section{ANTIMATTER}

Each type of charged particle of matter has an oppositely charged counterpart. These identical but oppositely charged particles are antimatter (or antiparticles). For example, the antimatter counterpart of a muon (negatively charged) is an antimuon (positively charged). Neutrinos do not have charge, but they also have antiparticles; understanding the nature of these antineutrinos is one of the most important mysteries in physics.

\section{NEUTRINO}

\section{FLAVORS}

Neutrinos come in three flavors, or types, named after different types of charged particles they produce when they interact with ordinary matter. The three fundamental negatively charged particles are the electron, the muon, and the tau particle. They are identical in every way except that the muon is 200 times heavier than the electron, and the tau is 3,500 times heavier. will pass through every second. Most of these mysterious particles move right through without making a sound or leaving a trace. But occasionally, very rarely, like once every ten-million trillion times, a neutrino will strike one of the atoms that make up a water molecule. That rare interaction may generate a tiny flash of light or leave some free electrical charge.

This flash is far too faint for your eyes to see. But scientists can build very sensitive detectors to pick up those faint signals. Like something out of Ghostbusters, the electronics inside the detectors amplify the signals to make the invisible "ghosts" visible!

But waiting for a "one-in-ten-million-trillion" neutrino interaction to take place can take a very long time. To catch enough neutrinos to learn anything about them, scientists need a bigger "glass of water"-huge tanks filled with millions of gallons! And they have to put the huge detector tanks deep underground. Why? Because neutrinos are not the only particles zipping down to Earth. Many other energetic particles known as cosmic rays also stream from outer space. Each one of those cosmic ray particles can cause a visible interaction in the detectors. If the detector tank was above ground, millions of cosmic ray interactions would easily drown out the rare neutrino signals. But with the detector deep underground, cosmic rays are stopped by interactions with Earth's atoms while neutrinos pass right through to leave their mark in the detector.

\section{DIFFERENT NEUTRINO DETECTORS AND NEUTRINO TYPES}

Neutrinos have a huge range of energies. That variation can lead to either a tiny flash of light or a very bright flash in water-filled detectors. Neutrinos also come in three different types, called flavors. Scientists have learned how to build detectors suited for each type of neutrino and their huge range of energies.

The interaction of each different type of neutrino produces a different kind of negatively charged particle. An electron neutrino produces an electron (simple enough, right?). The other two neutrino types are named for different, heavier subatomic particles: a muon neutrino produces a muon particle; and a tau neutrino produces a tau particle.

There are also antimatter versions of the three neutrino flavors. These are exactly the same as the ordinary-matter neutrinos, except they produce positively charged particles in the detector.

Scientists can tell these particles apart because each type leaves a different pattern in the detector. For example, muons leave straight tracks, electrons look like showers, and tau particles decay quickly to 
Figure 2

A scientist stands inside the ProtoDUNE detector at Europe's CERN laboratory before it was filled with cold $\left(-186^{\circ} \mathrm{C}\right.$ !) pure liquid argon. The scientist is inspecting the inside surface, which is in an insulated box made of very clean stainless steel. When the detector is filled and operating, these surfaces are energized with high voltage to cause charged particles produced by ionization of the liquid argon to drift and get picked up by sensitive electronics so scientists can record them. Credit: Fermi National Accelerator Laboratory.

\section{LIQUID ARGON}

Argon is an inert gas that forms one percent of Earth's atmosphere. It becomes liquid at $-186^{\circ} \mathrm{C}$ and has the clear appearance of water. It is good for detecting neutrinos because ionization lingers long enough to be detected.

\section{IONIZATION}

Ionization occurs when energetic particles knock electrons off atoms. The results are positively charged ions and free electrons.

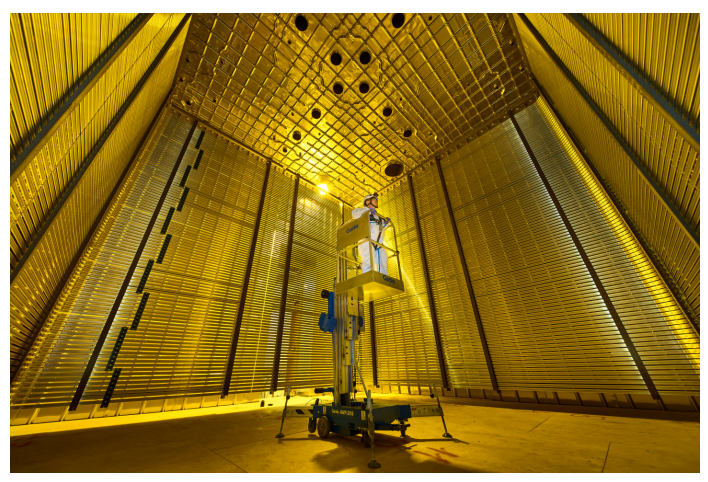

Figure 2

produce multiple straight tracks. Using the different patterns, scientists can decode the neutrino flavors and the hidden message carried by each ghostlike particle.

\section{SENSITIVE ELECTRONICS TAKE 3-D PICTURES}

A team of scientists is currently building a giant new neutrino detector deep underground in South Dakota in the United States. This experiment is known as the Deep Underground Neutrino Experiment (DUNE). Its detector will be filled with liquid argon.

Argon gas makes up only $1 \%$ of our atmosphere, but scientists can collect it to make very pure liquid argon. This super cold liquid $\left(-186^{\circ} \mathrm{C}\right)$ makes it easy to detect the charged particle pathways produced by neutrino interactions. That is because as fast charged particles move through the liquid argon they ionize the argon atoms in their path. That ionization allows the tracks they leave to show up like "lightning bolts."

But building a cold liquid argon detector is a big challenge. To make sure this new design will work, the international team recently built and tested a smaller version, known as ProtoDUNE (see Figure 2). They shot different types of particles through this detector at the European particle physics laboratory at the European council for Nuclear Research (CERN). Carefully designed electronics picked up and recorded the signals and computers transformed those measurements into beautiful 3-D images of the particle tracks (see Video 1)-like a five-megapixel digital camera that works in three dimensions!

\section{MESSENGERS FROM THE SUN, THE ATMOSPHERE, REACTORS, AND ACCELERATORS}

In the last 50 years, scientists have gotten better at detecting neutrinos. They have measured them coming from the sun, from collisions of cosmic rays with atoms in Earth's atmosphere, and from nuclear 
Figure 3

The four sources of neutrinos used for experiments. (A) The sun produces electron neutrinos $\left(v_{\mathrm{e}}\right)$. (B) Neutrinos of two types, muon $\left(v_{\mu}\right)$ and electron $\left(\nu_{\mathrm{e}}\right)$, are produced by collisions of high energy cosmic rays with atoms in Earth's atmosphere. (C) Nuclear reactors emit anti-electron neutrinos (anti $v_{\mathrm{e}}$ ). (D)

High-energy proton accelerators produce a beam of muon neutrinos $\left(\nu_{\mu}\right)$ that is directed through Earth Detectors are placed at different distances from the sources. Credit:

Tiffany Bowman, Brookhaven National Laboratory.

\section{VIDEO 1}

This image shows the tracks of many cosmic ray particles going through the protoDUNE detector. These data were used to test the detector performance. The colors correspond to the intensity of ionization in the liquid argon (red is less, and blue is more intense).

The ionization is moved by applying a powerful electric field to the detector. This 3-D image was created by analyzing and combining the electronic signals from thousands of wires on the walls that act like pixels in a digital camera. Credit: Chao Zhang, Brookhaven National Laboratory. You can view this data in 3 dimensions at this link.

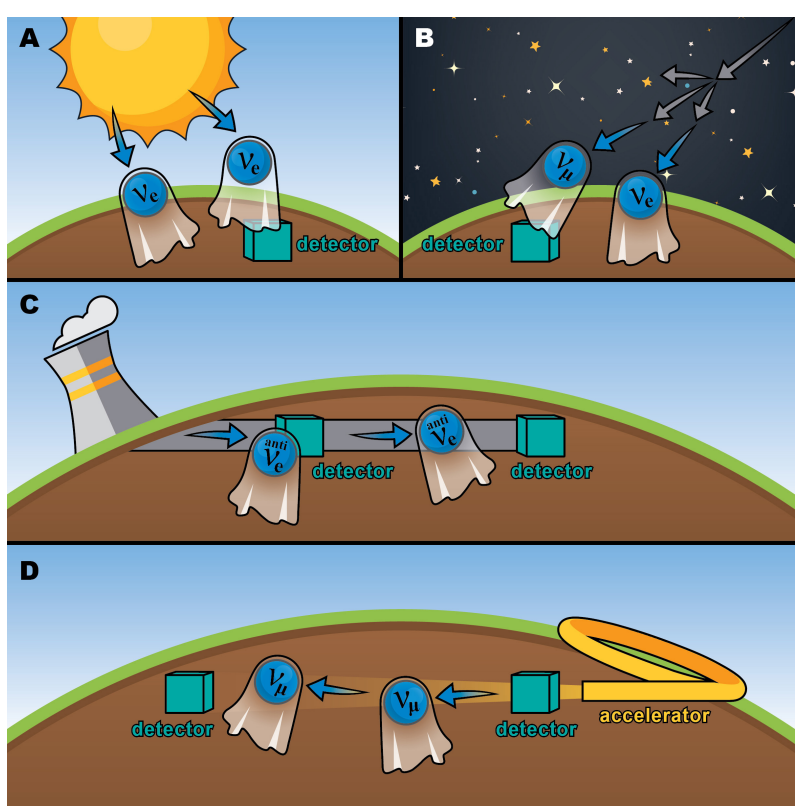

Figure 3

reactors that produce electricity (see Figure 3). They have also learned to make very energetic neutrinos at high-power particle accelerators. Each kind of source produces different neutrino flavors at different energies. By carefully counting neutrinos of each flavor at various distances from these sources, scientists have made two remarkable discoveries about neutrinos. First, they discovered that neutrinos of the three different types morph into each other as they travel through space! In other words, the messages the neutrinos carry change as they fly. Second, they discovered that neutrinos have a very tiny mass! Each neutrino particle weighs $<1$ millionth the mass of an electron.

The very first experiment that successfully detected solar neutrinos, by Brookhaven National Laboratory's Raymond Davis in the 1960's, found a surprise: Many of the neutrinos that scientists were expecting to detect were somehow "missing." But it was not because the detector was not working. The scientists had designed their detector to receive only one type of neutrino-electron neutrinos generated in the core of the sun. They could calculate exactly how many electron neutrinos should be detected. But only about one-third of the expected electron neutrinos showed up in the detector. Through follow-up experiments, scientists eventually learned the reason behind this mystery: Some of the electron neutrinos had morphed into one of the other two flavors (muon or tau) while on their journey from the sun. Because the detector was blind to those other two flavors, they appeared to be missing!

Davis's discovery of the "solar neutrino deficit" was, at first, very controversial. It had to be tested by other experiments. Other 


\section{ACCELERATORS}

Accelerators are machines in which charged particles, such as electrons or protons are sped up to nearly the speed of light. Scientists can collide very intense beams of such particles with targets to produce other subatomic particles, including energetic neutrinos.

\section{THE BIG BANG}

The Big Bang is shorthand for the event that started the universe from a single point filled with a hot, dense state of matter some 14 billion years ago. All the particles and matter we see today, including all the galaxies, stars, planets and more, formed as this point-like state expanded and cooled.

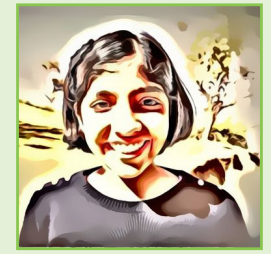

experiments used atmospheric neutrinos, reactor neutrinos, and accelerator neutrinos. In all of these experiments, scientists found neutrino flavors were "disappearing" - turning into other flavors over a long distance.

The new DUNE experiment in the United States and the HyperKamiokande experiment in Japan will reveal more details about the shape-shifting behavior of neutrinos and antineutrinos. If scientists find a difference between how neutrinos and antineutrinos transform, it might solve one of the most important mysteries in the universe: why the universe is made only of matter and not antimatter.

Scientists think both matter and antimatter were created in equal amounts in the Big Bang. Equal amounts of these two opposites should have destroyed one another leaving only light! So, the existence of only matter today is evidence that there was a small excess of matter. It is possible that the difference between neutrinos and antineutrinos caused this small excess as the universe expanded and cooled. If this is true, we have neutrinos to thank for the universe we have today, filled with all the visible stuff around us, including rocks, plants, animals, and people!

SUBMITTED: 21 October 2019; ACCEPTED: 24 March 2020;

PUBLISHED ONLINE: 07 May 2020.

EDITED BY: Joey Shapiro Key, University of Washington Bothell, United States

CITATION: Diwan MV and McNulty Walsh K (2020) Detecting Ghostlike Neutrinos: Tiny Messengers From the Universe. Front. Young Minds 8:45. doi: 10.3389/frym. 2020.00045

CONFLICT OF INTEREST: The authors declare that the research was conducted in the absence of any commercial or financial relationships that could be construed as a potential conflict of interest.

COPYRIGHT () 2020 Diwan and McNulty Walsh. This is an open-access article distributed under the terms of the Creative Commons Attribution License (CC BY). The use, distribution or reproduction in other forums is permitted, provided the original author(s) and the copyright owner(s) are credited and that the original publication in this journal is cited, in accordance with accepted academic practice. No use, distribution or reproduction is permitted which does not comply with these terms.

\section{YOUNG REVIEWERS}

\section{ANUSHKA, AGE: 12}

Hello, my name is Anushka. I have many hobbies like reading fiction, singing, and swimming. I like to have fun with my friends. I am interested in becoming a scientist when I grow up. 

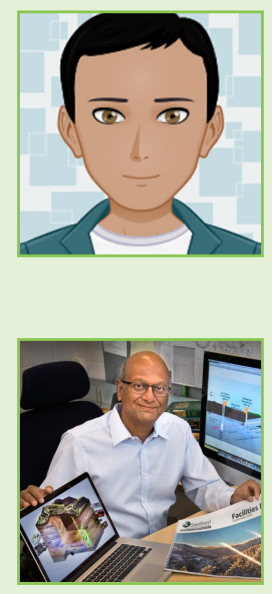

\section{RIK, AGE: 13}

My name is Rik and I am 13 years old. I guess that tells you I am a math nerd. Learning the guitar, playing soccer, and acting are also some of my favorite things to do.

\section{AUTHORS}

\section{MILIND VAMAN DIWAN}

Milind Diwan grew up playing marbles with his friends under a tamarind tree in India. The huge tree was rumored to be a house of ghosts. After coming to America, Diwan became interested in physics and went to graduate school at Brown University to earn a Ph.D. researching ghost-like neutrinos. He is now a senior physicist at Brookhaven Lab. He has worked on many particle physics experiments and detectors around the world. He pioneered the ideas that led to the DUNE project that will send an intense beam of neutrinos across the United States to a huge underground detector. *diwan@ibnl.gov

\section{KAREN MCNULTY WALSH}

Karen McNulty Walsh always loved learning about science, but she did not want to be a scientist. After studying biology at Vassar College, she went to NYU's Science, Health, and Environmental Reporting Program to become a science writer. She spent 10 years writing science articles for children-mostly for Scholastic's Science World magazine (read in classrooms) and Zillions (a kid's version of Consumer Reports that reviewed toys, snack foods, and other kids' products). Now she writes about the research at Brookhaven National Laboratory, and loves sharing her excitement as she learns about superconductors, subatomic particles, batteries, biofuels, and more. 